\title{
Design and Construction Processes of Carbon Monoxide Leak Detection System for Domestic Application
}

\author{
M.A. Mohtadi Bonab \\ Bonab Higher Education Complex \\ E-mail: mohtadibonab@bonabetu.ac.ir \\ R.Karimdadashi \\ Bonab Higher Education Complex \\ E-mail: karimdadashi@bonabetu.ac.ir \\ Gh. Jabbari \\ Institute of Energy and Hydro Technology \\ E-mail: gh.Jabbari@ieht.ac.ir
}

Accepted: December 27, 2010

doi:10.5539/mas.v5n2p161

\begin{abstract}
In this paper, design and construction processes of a detector have been presented. $\mathrm{SnO}_{2}$ semiconductor sensor has been used at this detector. It has been made in laboratory with vacuum equipment by using CVD method and a heater and a stabilizer circuit of temperature have been used for increasing the sensor temperature and making stable at this temperature. An amplifier circuit coupled to the gas sensor. Also a signal processing circuit and an 8051 programmable micro controller has been used for detection.
\end{abstract}

Keywords: Sensor, Semiconductor, Leak detection, Micro controller

Introduction

Carbon monoxide (CO) is a colorless, odorless, tasteless, toxic gas produced during the incomplete combustion of fuels. Carbon monoxide is rapidly absorbed by the lungs and quickly passes to the blood. It is able to bind to the red blood cells and prevent them from delivering oxygen to the body tissues. Carbon monoxide has a three fourth hour half-life; this means after breathing carbon monoxide, three fourth hour of breathing fresh air eliminates only half the $\mathrm{CO}$ from the blood. Therefore, a system that is able to detect the carbon monoxide will be useful to domestic operation (Cobb N, Entzel RA, 1988).

In this paper, design and construction processes of a detector have been presented. $\mathrm{SnO}_{2}$ semiconductor sensor has been used at this detector which has variable electrical characteristics at high temperatures in vicinity of hydrocarbon gases. It has been made in laboratory with vacuum equipment by using CVD method.

Experimental results with this sensor show that the most sensibility for Co gas will be at $190^{\circ} \mathrm{C}$. A heater and a stabilizer circuit of temperature have been used for detection part increasing the temperature up to $190^{\circ} \mathrm{C}$ and making stable at this temperature.

A 8051 programmable micro controller has been used for detection. It has been programmed in a manner that it's possible to define the supposed level of Co gas concentration so that the detector will operate at this level.

The main advantage of this apparatus is its flexibility so that with changing the temperature of sensor and defining various concentrations, it can be used for detecting of different gases.

\section{Sensor construction}

The method which has used in this project consists of cool rim, low pressure and bottom hot layer(H. Wan Cheong et. al. 1991). The schematic of CVD system which is used for making a tin oxide layer has shown in figure 1.

In order to make $\mathrm{SnO}_{2}$ sensors on the glass, at first the surface of the glass was cleaned with the acid, asten, alcohol and water. This cleaning ensures that the surface of the glass has not any dirt. Then the glass is placed on the mica layer. (E) We must ensure that no leakage will occur. Vacuum pump will be started to work and we wait approximately half hour until inside pressure of the housing is reached to suitable magnitude for layer, and the inside vacuum is reached to stable condition. Then the electrical current is passed from the fileman until the temperature of the glass is reached to predetermined level. The temperature range of 320-450 that is suitable for $\mathrm{SnO}_{2}$ layer (S. G. Ansari, S. W. Gosavi, S. A. Gangal, 1997). After holding the temperature at the 400 degree, the vapors are entered to housing A. The vapors are tin coloreid. Because of the high temperature of the inside housing, The $\mathrm{HCl}$ gas is exit from $\mathrm{F}$ outlet and the tin oxide particles become sediment on the glass lams and act as a sensor. 


$$
\mathrm{SnCl}_{4}+2 \mathrm{H}_{2} \mathrm{O} \longrightarrow \mathrm{SnO}_{2}+4 \mathrm{HCl}(\mathrm{g})
$$

To make other prototypes such as iridium, we can put it in the impure source, and the weight percentages of $\mathrm{InCl} 3$ soluble and try the test such as first test with different weight percentages. But in this stage we must combine $\mathrm{SnCl} 4$ and $\mathrm{InCl} 3$ vapors outside the housing and then the combined vapor must be directed to vacuum housing. The relation (2) will be available and created gases will go out through vacuum. Fig. 3 shows that how electrodes are placed on sensors.

$$
2 \mathrm{InCl}_{3}+3 \mathrm{H}_{2} \mathrm{O} \longrightarrow \mathrm{In}_{2} \mathrm{O}_{3}(\mathrm{~s})+6 \mathrm{HCl}(\mathrm{g})
$$

\section{Determination of a suitable temperature for leak detecting of $\mathrm{Co}$}

Because the resistance of the semi- conductor sensors varies with the temperature variations, we have to fix the temperature at the particular temperature. In this case, the resistance variations will be available only due to existence of $\mathrm{CO}$ gas and other elements will not affect this resistance. In addition, because sensor sensitivity in various temperatures varies for several gases, we must obtain the suitable temperature in order to have a highest sensitivity to CO and must fix this temperature during leak detection process (A. Salehi, 1998). For sensitivity calculation the following relation is used.

$$
S=\left(\Delta R / R_{a}\right) \times 100=\left(R_{a}-R_{g}\right) \times 100 / R_{a}
$$

In this relation $\mathrm{Ra}$ is sensor resistance in environmental condition and $\mathrm{Rg}$ is the resistance of the sensor in the gas environment.

The samples have been put in housing that its temperature was completely controllable. The tests have don in temperatures between 50-250 degrees. By entering some amount of this gas in various temperatures to the housing, the sensitivity of gas in given temperature and consistency is determined. In figure 1-3 the sensor sensitivity curve to Co in various temperatures has shown. In that case, the consistency of the gas is $1000 \mathrm{ppm}$.

From figure 1-3 it is obvious that with the increasing of temperature, the sensitivity also increases and at the temperatures between 150-200 degree the maximum sensitive is reached to 15.4. After this temperature range, the sensitivity is decreased. Figure 3 shows the maximum sensitivity at 175 degree.After determining the suitable temperature for measuring the $\mathrm{Co}$, we need to design a suitable electrical circuit with the help of electrical resistance variation at the adjacent of Co gas, the leak detection process will be occurred.

\section{Circuit design}

In order to control the sensor output, one microcontroller has used and after the possessing of the input information, the output is transmitted to LCD unit.

As shown in Fig.5., one NTS and start/stop circuit is used to fix the temperature.

\section{System algorithm}

The microcontroller program such as this algorithm has been written in which at first by the use of STORE button the appropriate magnitude of leak detection is entered to system. And when the consistency of the gas is enough to bring the resistance of the system to entered magnitude, the system is started to make an alarm.

\section{References}

A. Salehi. (1998). The Effects of Deposition Ratio and Substrate Temperature of ITO Thin Films on Electrical and Optical Properties, Thin Solid Films, 324 (1998) 214-218.

Cobb N, Entzel RA. (1991). Unintentional carbon monoxide-related deaths in the united states. 1979 through 1988. JAMA 1991:266:659-63

H. Wan Cheong et. al. (1991). The Role of Additive in $\mathrm{Sno}_{2}$ Semiconductor Gas Sensors, IEEE Trans., Semiconductor Devices, 1991, pp.154-156.

S. G. Ansari, S. W. Gosavi, S. A. Gangal. (1997). Characterization of $\mathrm{Sno}_{2}-$ Based $\mathrm{H}_{2}$ gas Sensors Fabricated by Different deposition Techniques, J.Mater SCI Mater. Elec. 8(1997)23-27.

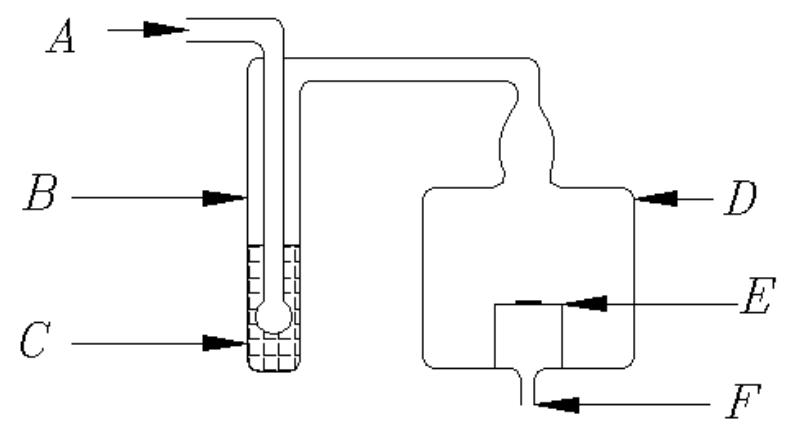

Figure 1. Schematic of CVD system 


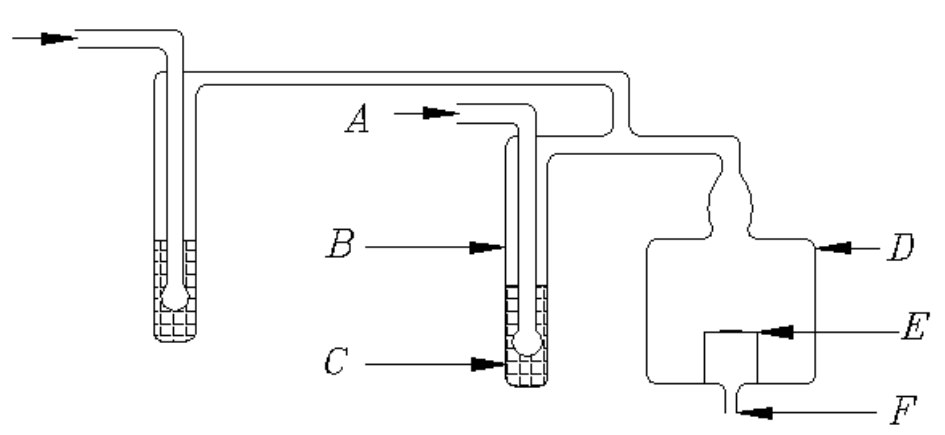

Figure 2. Method of adding the iriduim impurity to sensor

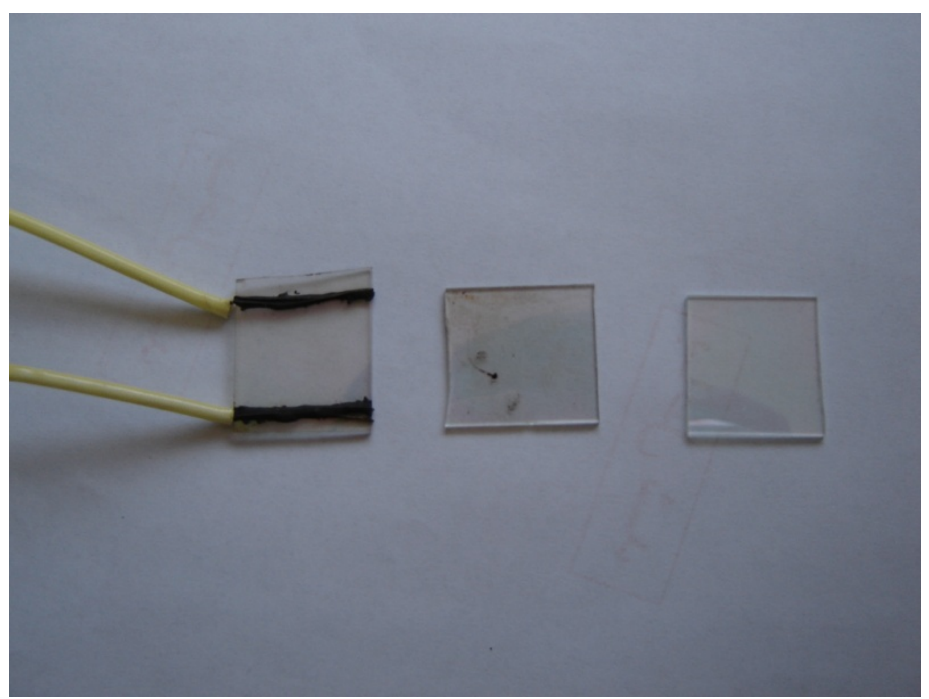

Figure 3. Placing electrodes on sensors

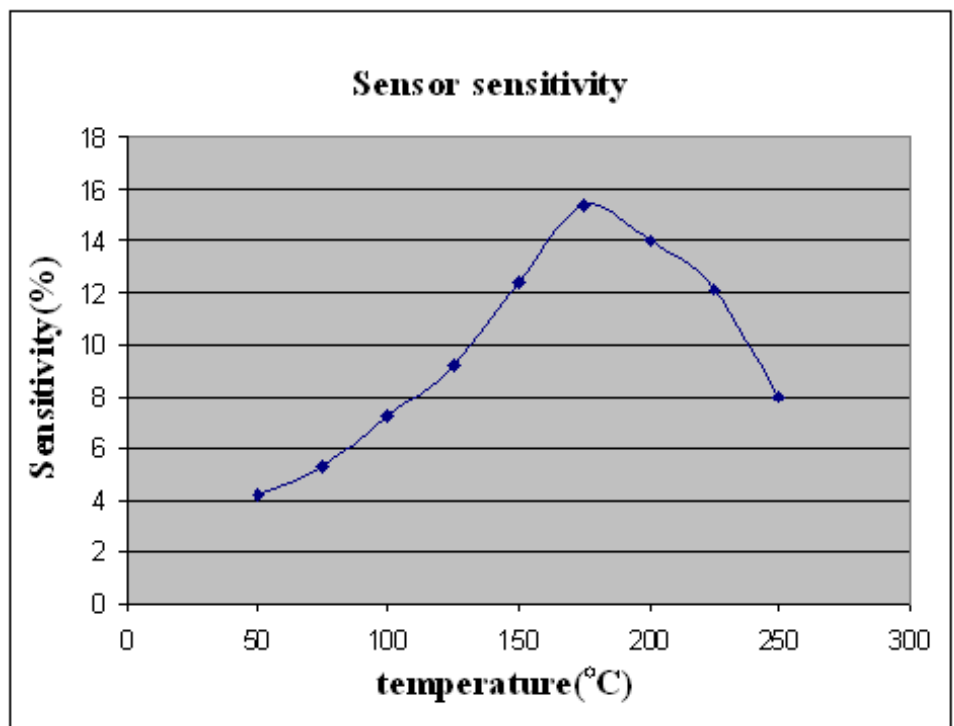

Figure 4 . The sensitivity curve of sensor at various temperatures 


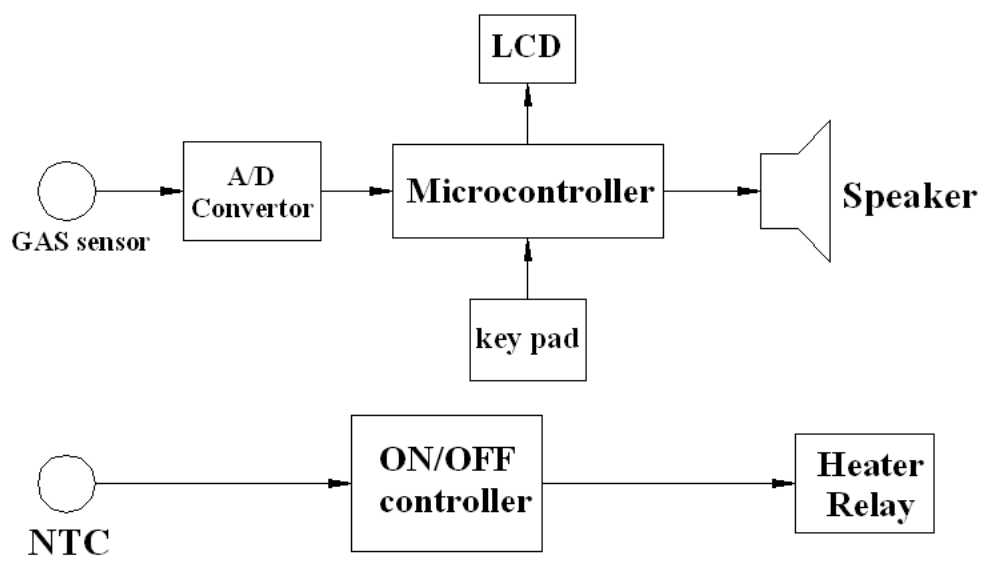

Figure 5. Electrical circuit of the system

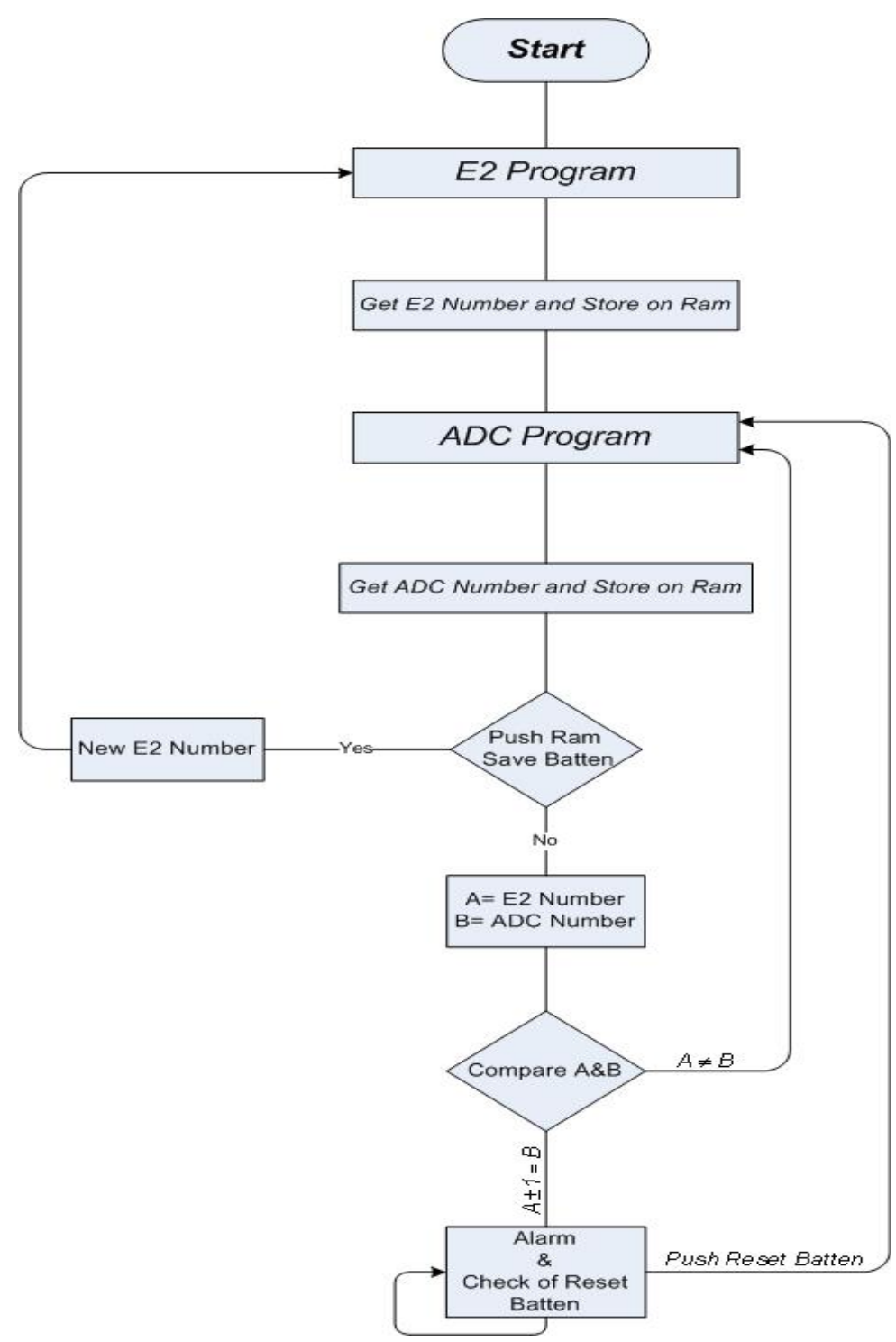

Figure 6. System algorithm 